\title{
ET - platinum therapy comes home
}

Apart from immunotherapy, little treatment progress is evident for wild-type EGFR or ALK non-small-cell lung cancer (NSCLC). At Most patients receive doublet platinum-based chemotherapy, for which effective predictive biomarkers are lacking. The excision repair cross complementing group 1 (ERCC1) protein is a potential treatment biomarker: its expression has been associated with platinum resistance in retrospective studies. This finding prompted the prospective, randomized phase III ERCC1 trial (ET) to evaluate the predictive and prognostic utility of ERCC1 in 648 patients treated with platinum or nonplatinum therapy.

Patients with advanced-stage squamous NSCLC treated with non-platinum-based chemotherapy had a significantly worse response rate, progression-free and overall survival than those who received platinumbased chemotherapy. However, ERCC1 was neither prognostic nor predictive for survival outcomes. Jean-Charles Soria, who wrote an accompanying editorial comments: "the findings of the ET trial reinforce that platinum-based chemotherapy should remain the cytotoxic treatment backbone in squamous
NSCLC. The results also confirm the daunting absence of predictive companion biomarkers for selecting patients who will derive benefit from platinum-based therapy - the most frequently prescribed first-line treatment of NSCLC worldwide. Immunohistochemistry testing methods are currently unable to reliably assess ERCC1 functionality."

Beyond the exploration of specific mutations, genomic signatures, or gene-expression patterns, the identification and quantification of proteins expressed by tumour cells is a promising field of investigation for ERCC1 and other DNA-repair proteins. "Once identified and validated, the development of the biomarker and its assay should be aimed at making this technology available to the largest number of patients, notably by using liquid biopsies and affordable techniques on tissue-samples," Soria concludes.

Lisa Hutchinson

ORIGINAL ARTICLE Lee, S. M. et al. Randomized prospective biomarker trial of ERCC 1 for comparing platinum and nonplatinum therapy in advanced non-small-cell lung cancer: ERCC1 Trial (ET).J. Clin. Oncol. http://dx.doi.org/10.1200/ ICO.2016.70.5053 (2016) 